\title{
INSTABILITY OF WEIGHTED COMPOSITION OPERATORS BETWEEN SPACES OF CONTINUOUS FUNCTIONS
}

\author{
JESÚS ARAUJO AND JUAN J. FONT
}

\begin{abstract}
Let $\epsilon>0$. A continuous linear operator $T: C(X) \longrightarrow$ $C(Y)$ is said to be $\epsilon$-disjointness preserving if $\|(T f)(T g)\|_{\infty} \leq \epsilon$, whenever $f, g \in C(X)$ satisfy $\|f\|_{\infty}=\|g\|_{\infty}=1$ and $f g \equiv 0$. In this paper we address basically the following question:

How far can the set of weighted composition operators be from a given $\epsilon$-disjointness preserving operator?

We provide sharp instability bounds.
\end{abstract}

\section{INTRODUCTION}

Let $\mathbb{K}$ denote the field of real or complex numbers. Let $C(X)$ stand for the Banach space of all $\mathbb{K}$-valued continuous functions defined on a compact Hausdorff $X$ and equipped with its usual supremum norm.

An operator $S: C(X) \longrightarrow C(Y)$ is said to be a weighted composition map if there exist a function $a \in C(Y)$ and a map $h: Y \longrightarrow X$, continuous on $c(a):=\{y \in Y: a(y) \neq 0\}$, such that

$$
(S f)(y)=a(y) f(h(y))
$$

for every $f \in C(X)$ and $y \in Y$.

We include the case when $S \equiv 0$ as a weighted composition map (being $c(a)=\emptyset$ ). Obviously every weighted composition map is linear and continuous, and is also disjointness preserving, in the sense that given $f, g \in C(X), f g \equiv 0$ yields $(S f)(S g) \equiv 0$. Reciprocally, it is well known that a continuous disjointness preserving operator is a weighted composition (see for instance [6], [5], [7])).

Given $\epsilon>0$, a continuous linear operator $T: C(X) \longrightarrow C(Y)$ is said to be $\epsilon$-disjointness preserving if $\|(T f)(T g)\|_{\infty} \leq \epsilon$, whenever

2000 Mathematics Subject Classification. Primary 47B38; Secondary 46J10, 47B33.

Research of the first author was partially supported by the Spanish Ministry of Science and Education (Grant number MTM2006-14786).

Research of the second author was partially supported by the Spanish Ministry of Science and Education (Grant number MTM2008-04599), and by Bancaixa (Projecte P1-1B2008-26). 
$f, g \in C(X)$ satisfy $\|f\|_{\infty}=\|g\|_{\infty}=1$ and $f g \equiv 0$ (or, equivalently, if $\|(T f)(T g)\|_{\infty} \leq \epsilon\|f\|_{\infty}\|g\|_{\infty}$ whenever $\left.f g \equiv 0\right)$.

In [4] G. Dolinar studied when an $\epsilon$-disjointness preserving operator is close to a weighted composition map, and proved that, given $\epsilon>0$ and an $\epsilon$-disjointness preserving operator $T: C(X) \longrightarrow C(Y)$ with $\|T\|=1$, there exists a weighted composition map $S: C(X) \longrightarrow C(Y)$ such that

$$
\|T-S\| \leq 20 \sqrt{\epsilon}
$$

This bound was recently sharpened to $\sqrt{17 \epsilon / 2}$ in [1], where it was also proved, by means of an example, that this new bound cannot be improved.

In this paper we address what could be regarded as the reverse question. Namely, we study how far apart an $\epsilon$-disjointness preserving operator can be from the set of all weighted composition operators.

In general, we prove that the answer does not depend on the topological features of the space $X$ but on its cardinality (denoted by card $X$ ). If we assume that $Y$ has at least two points, then the number $2 \sqrt{\epsilon}$ is a valid bound if $X$ is infinite (Theorem 2.1). A different value plays the same rôle if $X$ is finite (Theorem 3.1).

We also prove that these estimates are sharp in every case (Theorems 2.2 and 3.2). Indeed, instead of providing a concrete counterexample, we show that these bounds are the best for a general family of spaces $Y$, namely, whenever $Y$ consists of the Stone-Čech compactification of any discrete space.

Notation. Throughout $\mathbb{K}=\mathbb{R}$ or $\mathbb{C} . \quad X$ and $Y$ will be compact Hausdorff spaces with at least two points (when $X$ has just one point we obtain a trivial case, and when $Y$ consists of a single point, we are dealing with functionals, and the results take a completely different form, as can be seen in [2]).

Given a compact Hausdorff space $Z, C(Z)^{\prime}$ will denote the space of linear and continuous functionals defined on $C(Z)$. For $\varphi \in C(Z)^{\prime}$, we will write $\lambda_{\varphi}$ to denote the measure which represents it. Also, for $x \in Z$, $\delta_{x}$ will be the evaluation functional at $x$, that is, $\delta_{x}(f):=f(x)$ for every $f \in C(Z)$, and given $T: C(X) \longrightarrow C(Y)$ linear and continuous, we set $T_{y}:=\delta_{y} \circ T$ for each $y \in Y$.

For $f \in C(Z), 0 \leq f \leq 1$ means that $f(x) \in[0,1]$ for every $x \in$ $Z, c(f)=\{x \in Z: f(x) \neq 0\}$ denotes its cozero set and $\operatorname{supp}(f)$ its support. 
We denote by $\epsilon-\mathbf{D P}(X, Y)$ the set of all $\epsilon$-disjointness preserving operators from $C(X)$ to $C(Y)$, and by $\mathbf{W C M}(X, Y)$ the set of all weighted composition maps from $C(X)$ to $C(Y)$.

In a Banach space $E$, for $e \in E$ and $r>0, B(e, r)$ and $\bar{B}(e, r)$ denote the open and the closed balls of center $e$ and radius $r$, respectively.

\section{The CASE WHEn $X$ IS Infinite}

Our first result shows that the bound depends on whether or not the space $X$ admits a continuous measure (recall that a Borel measure on a Hausdorff space is said to be continuous if it vanishes on all singletons; see for instance [3, Definition 7.14.14]).

Theorem 2.1. Let $0<\epsilon<1 / 4$. Suppose that $X$ is infinite. Then for each $t<1$, there exists $T \in \epsilon-\mathbf{D P}(X, Y)$ with $\|T\|=1$ such that

$$
B(T, 2 t \sqrt{\epsilon}) \cap \mathbf{W C M}(X, Y)=\emptyset .
$$

Furthermore, if $X$ admits a continuous regular probability measure, then $T$ can be taken such that

$$
B(T, 2 \sqrt{\epsilon}) \cap \mathbf{W C M}(X, Y)=\emptyset .
$$

In Theorem 2.2, we see that the above bounds are sharp for some families of extremely disconnected spaces $Y$. This should be compared with [1, Example 4.6]), where the local connectedness of some other spaces $Y$ plays an important rôle when proving that their corresponding stability bounds are sharp and, consequently, far from sharp with respect to the instability bounds given above.

Theorem 2.2. Let $0<\epsilon<1 / 4$. Suppose that $Y$ is the Stone- $\check{C}$ ech compactification of a discrete space with at least two points, and that $X$ is infinite. Let $T \in \epsilon-\mathbf{D P}(X, Y)$ with $\|T\|=1$. Then

$$
\bar{B}(T, 2 \sqrt{\epsilon}) \cap \mathbf{W C M}(X, Y) \neq \emptyset \text {. }
$$

Furthermore, if $X$ does not admit a continuous regular probability measure and $Y$ is finite (with card $Y \geq 2$ ), then

$$
B(T, 2 \sqrt{\epsilon}) \cap \mathbf{W C M}(X, Y) \neq \emptyset .
$$

Proof of Theorem 2.1. For $\delta>0$, let us choose a regular Borel probability measure $\mu$ on $X$ such $\mu(\{x\}) \leq \delta / 2$ for every $x \in X$.

Next, fix $y_{0}, y_{1}$ in $Y$ and $x_{0} \in X$. After choosing two disjoint neighborhoods, $U\left(y_{0}\right)$ and $U\left(y_{1}\right)$, of $y_{0}$ and $y_{1}$, respectively, we define two continuous functions, $\alpha: Y \longrightarrow[0,2 \sqrt{\epsilon}]$ and $\beta: Y \longrightarrow[0,1]$, with the following properties:

$$
\text { - } \alpha\left(y_{0}\right)=2 \sqrt{\epsilon}
$$


- $\operatorname{supp}(\alpha) \subset U\left(y_{0}\right)$

- $\beta\left(y_{1}\right)=1$

- $\operatorname{supp}(\beta) \subset U\left(y_{1}\right)$

Next, for each $y \in Y$, we define two continuous linear functionals on $C(X)$ as follows:

$$
\begin{gathered}
F_{y}(f)=\beta(y) \delta_{x_{0}}(f) \\
G_{y}(f)=\alpha(y) \int_{X} f d \mu
\end{gathered}
$$

By using these functionals we can now introduce a linear map $T$ : $C(X) \longrightarrow C(Y)$ such that $(T f)(y)=F_{y}(f)+G_{y}(f)$ for every $f \in$ $C(X)$.

Let us first check that $\|T\|=1$. To this end, it is apparent that $(T \mathbf{1})\left(y_{1}\right)=F_{y_{1}}(\mathbf{1})+G_{y_{1}}(\mathbf{1})=1+0=1$, where $\mathbf{1}$ denotes the constant function equal to 1 . Consequently, $\|T\| \geq 1$. On the other hand, it is easy to see that if $f \in C(X)$ satisfies $\|f\|_{\infty}=1$, then $|(T f)(y)| \leq 1$ for every $y \in Y$. Hence, $\|T\|=1$.

The next step consists of checking that $T$ is $\epsilon$-disjointness preserving. Let $f, g \in C(X)$ with $\|f\|_{\infty}=\|g\|_{\infty}=1$ and such that $c(f) \cap c(g)=\emptyset$. It is easy to see that $(T f)(y)(T g)(y)=0$ whenever $y \notin U\left(y_{0}\right)$. On the other hand, if $y \in U\left(y_{0}\right)$, then $|(T f)(y)(T g)(y)|=\left|G_{y}(f)\right|\left|G_{y}(g)\right|$. It is clear that there exist two unimodular scalars $a_{1}, a_{2} \in \mathbb{K}$ such that $a_{1} G_{y}(f)=\left|G_{y}(f)\right|$ and $a_{2} G_{y}(g)=\left|G_{y}(g)\right|$. Since $\left\|a_{1} f+a_{2} g\right\|_{\infty}=1$, then

$$
\begin{aligned}
\left|G_{y}(f)\right|+\left|G_{y}(g)\right| & =G_{y}\left(a_{1} f+a_{2} g\right) \\
& =\alpha(y) \int_{X}\left(a_{1} f+a_{2} g\right) d \mu \\
& \leq \alpha(y)
\end{aligned}
$$

Consequently, $\left|G_{y}(f)\right|\left|G_{y}(g)\right| \leq \alpha(y)^{2} / 4$. Indeed,

$$
|(T f)(y)(T g)(y)|=\left|G_{y}(f)\right|\left|G_{y}(g)\right| \leq \frac{\alpha(y)^{2}}{4} \leq \frac{(2 \sqrt{\epsilon})^{2}}{4}=\epsilon
$$

Finally, we will see that $\|T-S\| \geq 2 \sqrt{\epsilon}(1-\delta)$ for every weighted composition map $S: C(X) \longrightarrow C(Y)$.

Let $S \in \mathbf{W C M}(X, Y)$, and let $h: c(S \mathbf{1}) \longrightarrow X$ be its associated map. It is clear that, if $(S \mathbf{1})\left(y_{0}\right)=0$, then $\|T-S\|=\mid(T-S)(\mathbf{1})\left(y_{0}\right) \|=$ $2 \sqrt{\epsilon}$, so we may assume that $y_{0}$ belongs to $c(S \mathbf{1})$. By the regularity of the measure $\mu$, there exists an open neighborhood $U$ of $h\left(y_{0}\right)$ such that $\mu(U)<\delta$. Let us select $f \in C(X)$ satisfying $0 \leq f \leq 1, f\left(h\left(y_{0}\right)\right)=0$, 
and $f \equiv 1$ on $X \backslash U$. Obviously $(S f)\left(y_{0}\right)=0$ and $\left|(T f)\left(y_{0}\right)\right|=\left|G_{y_{0}}(f)\right|$. Hence

$$
\begin{aligned}
\|T-S\| & \geq\left|(T f)\left(y_{0}\right)\right| \\
& \geq \alpha\left(y_{0}\right) \int_{X \backslash U} f d \mu \\
& \geq 2 \sqrt{\epsilon}(1-\delta) .
\end{aligned}
$$

This proves the first part. The second part is immediate because, being the measure of each point equal to zero, $\delta$ can be taken as small as wanted.

Proof of Theorem 2.2. We are assuming that there exists a discrete space $Z$ such that $Y=\beta Z$. Of course $Y$ may be finite (that is, $Y=Z$ ), and this is necessarily the case when we consider the second part of the theorem. Let $Z_{0}:=\left\{y \in Z:\left\|T_{y}\right\|>2 \sqrt{\epsilon}\right\}$, which is a nonempty closed and open subset of $Z$, and

$$
Z_{1}:=\left\{z \in Z \backslash Z_{0}: \exists x_{z} \in X \text { with }\left|\lambda_{T_{z}}\left(\left\{x_{z}\right\}\right)\right|>0\right\} .
$$

Fix any $x_{0} \in X$. By [1, Lemma 2.3], we can define a map $h: Z \longrightarrow X$ such that $\left|\lambda_{T_{z}}(\{h(z)\})\right| \geq \sqrt{\left\|T_{z}\right\|^{2}-4 \epsilon}$ for every $z \in Z_{0}$, and such that $h(z):=x_{z}$ for $z \in Z_{1}$, and $h(z):=x_{0}$ for $z \notin Z_{0} \cup Z_{1}$. Also, since $Z$ is discrete, then $h$ is continuous, and consequently it can be extended to a continuous map from $Y$ to $X$ (when $Y \neq Z$ ). We will denote this extension also by $h$.

Define $\alpha: Z \longrightarrow \mathbb{K}$ as $\alpha(z):=\lambda_{T_{z}}(\{h(z)\})$ if $z \in Z_{0} \cup Z_{1}$, and $\alpha(z):=$ 0 otherwise, and extend it to a continuous function, also called $\alpha$, defined on $Y$. Then consider $S: C(X) \longrightarrow C(Y)$ defined as $(S f)(y):=$ $\alpha(y) f(h(y))$ for every $f \in C(X)$ and $y \in Y$.

Let us check that $\|T-S\| \leq 2 \sqrt{\epsilon}$. Take $f \in C(X)$ with $\|f\|_{\infty} \leq 1$. First, suppose that $z \in Z \backslash\left(Z_{0} \cup Z_{1}\right)$. Then $(S f)(z)=0$, so

$$
|(T f)(z)-(S f)(z)|=|(T f)(z)| \leq 2 \sqrt{\epsilon} \text {. }
$$

Now, if $z \in Z_{1}$, then $\left\|T_{z}\right\| \leq 2 \sqrt{\epsilon}$ and, as in the proof of [1, Lemma $2.4]$

$$
|(T f)(z)-(S f)(z)| \leq\left\|T_{z}\right\|-\left|\lambda_{T_{z}}(\{h(z)\})\right|<2 \sqrt{\epsilon} .
$$

On the other hand, if $z \in Z_{0}$, we know by [1, Corollary 2.5] that

$$
|(T f)(z)-(S f)(z)| \leq\left\|T_{z}\right\|-\sqrt{\left\|T_{z}\right\|^{2}-4 \epsilon} .
$$

By $[1$, Lemma 3.4], we have $|(T f)(z)-(S f)(z)|<2 \sqrt{\epsilon}$ for every $z \in$ $Z_{0}$. By continuity, we see that the same bound applies to every point in $Y$, and the first part is proved. 
Finally, in the second case, that is, when $X$ does not admit a continuous regular probability measure and $Y$ is finite, we have that $Y=Z$, and that $Z \backslash\left(Z_{0} \cup Z_{1}\right)$ consists of those points satisfying $\left\|T_{z}\right\|=0$. The conclusion is then easy.

\section{The CASE When $X$ is FInite}

The best instability bounds in the finite case depend on the sequence $\left(\omega_{n}\right)$, where for each $n \in \mathbb{N}$,

$$
\omega_{n}:=\frac{n^{2}-1}{4 n^{2}} .
$$

These instability bounds are given in terms of the function $r_{X}$ : $(0,1 / 4) \longrightarrow \mathbb{R}$ (recall that we are assuming card $X \geq 2)$, defined as

$$
r_{X}(\epsilon):=\left\{\begin{aligned}
2 \sqrt{\frac{(n-1) \epsilon}{n+1}} & \text { if } n:=\operatorname{card} X \text { is odd and } \epsilon \leq \omega_{n} \\
\frac{n-1}{n} & \text { if } n:=\operatorname{card} X \text { is odd and } \epsilon>\omega_{n} \\
\frac{2(n-1) \sqrt{\epsilon}}{n} & \text { if } n:=\operatorname{card} X \text { is even }
\end{aligned}\right.
$$

Theorem 3.1. Let $0<\epsilon<1 / 4$. Suppose that $X$ is finite. Then there exists $T \in \epsilon-\mathbf{D P}(X, Y)$ with $\|T\|=1$ such that

$$
B\left(T, r_{X}(\epsilon)\right) \cap \mathbf{W C M}(X, Y)=\emptyset .
$$

The next result (Theorem 3.2) says that Theorem 3.1 provides a sharp bound, and gives a whole family of spaces $Y$ for which the same one is a bound for stability as well.

Theorem 3.2. Let $0<\epsilon<1 / 4$. Suppose that $Y$ is the Stone-Cech compactification of a discrete space with at least two points, and that $X$ is finite. Let $T \in \epsilon-\mathbf{D P}(X, Y)$ with $\|T\|=1$. Then

$$
\bar{B}\left(T, r_{X}(\epsilon)\right) \cap \mathbf{W C M}(X, Y) \neq \emptyset \text {. }
$$

Proof of Theorem 3.1. We first prove the result when $n$ is odd. We follow the same ideas and notation as in the proof of Theorem 2.1, with some differences. Namely, we directly take $\mu(\{x\})=1 / n$ for every $x \in X$, and use a new function

$$
\alpha: Y \longrightarrow\left[0, \min \left\{\frac{2 n \sqrt{\epsilon}}{\sqrt{n^{2}-1}}, 1\right\}\right]
$$

such that

$$
\alpha\left(y_{0}\right)=\min \left\{\frac{2 n \sqrt{\epsilon}}{\sqrt{n^{2}-1}}, 1\right\}
$$


and $\operatorname{supp}(\alpha) \subset U\left(y_{0}\right)$. Notice that $\alpha\left(y_{0}\right)=2 n \sqrt{\epsilon} / \sqrt{n^{2}-1}$ if $\epsilon \leq \omega_{n}$, and $\alpha\left(y_{0}\right)=1$ otherwise.

Clearly $\|T\|=1$, and using the fact that

$$
\frac{(n-1)(n+1)}{4 n^{2}}=\max \left\{\frac{l(n-l)}{n^{2}}: 0 \leq l \leq n\right\},
$$

we easily see that $T$ is $\epsilon$-disjointness preserving both if $\epsilon \leq \omega_{n}$ and if $\epsilon>\omega_{n}$. On the other hand, by the definition of the measure, reasoning as in the proof of Theorem 2.1, we easily check that $\|T-S\| \geq$ $(1-1 / n) \alpha\left(y_{0}\right)$ for every weighted composition $S$.

Finally, we follow the above pattern to prove the result when $n$ is even. In particular we also take $\mu(\{x\})=1 / n$ for every $x \in X$, and use a function $\alpha: Y \longrightarrow[0,2 \sqrt{\epsilon}]$ with $\alpha\left(y_{0}\right)=2 \sqrt{\epsilon}$ and $\operatorname{supp}(\alpha) \subset U\left(y_{0}\right)$. The rest of the proof follows as above.

We shall need the following proposition.

Proposition 3.3. Let $0<\epsilon<1 / 4$. Suppose that $X$ is a finite set of cardinality $k \in 2 \mathbb{N}$. If $\varphi \in \epsilon-\mathbf{D P}(X, \mathbb{K})$ and $\|\varphi\|=1$, then there exists $x \in X$ such that

$$
\left|\lambda_{\varphi}(\{x\})\right| \geq \frac{1+\sqrt{1-4 \epsilon}}{k} .
$$

Proof. By [1, Lemma 2.2], we can assume without loss of generality that $\varphi$ is positive. Suppose that $k=2 m, m \in \mathbb{N}$. Notice that there cannot be $m$ different points $x_{1}, \ldots, x_{m} \in X$ with

$$
\lambda_{\varphi}\left(\left\{x_{i}\right\}\right) \in\left(\frac{1-\sqrt{1-4 \epsilon}}{k}, \frac{1+\sqrt{1-4 \epsilon}}{k}\right)
$$

for every $i \in\{1, \ldots, m\}$, because otherwise

$$
\lambda_{\varphi}\left(\left\{x_{1}, \ldots, x_{m}\right\}\right) \in\left(\frac{1-\sqrt{1-4 \epsilon}}{2}, \frac{1+\sqrt{1-4 \epsilon}}{2}\right),
$$

against [1, Lemma 2.1]. This implies that there exist at least $m+1$ points whose measure belongs to

$$
\left[0, \frac{1-\sqrt{1-4 \epsilon}}{k}\right] \cup\left[\frac{1+\sqrt{1-4 \epsilon}}{k}, 1\right] .
$$

Suppose that at least $m$ different points $x_{1}, \ldots, x_{m} \in X$ satisfy $\lambda_{\varphi}\left(\left\{x_{i}\right\}\right) \leq$ $(1-\sqrt{1-4 \epsilon}) / k$. Then $\lambda_{\varphi}\left(\left\{x_{1}, \ldots, x_{m}\right\}\right) \leq(1-\sqrt{1-4 \epsilon}) / 2$, and consequently we have that $\lambda_{\varphi}\left(X \backslash\left\{x_{1}, \ldots, x_{m}\right\}\right) \geq(1+\sqrt{1-4 \epsilon}) / 2$. Since $X \backslash\left\{x_{1}, \ldots, x_{m}\right\}$ has $m$ points, this obviously implies that there exists $x \in X \backslash\left\{x_{1}, \ldots, x_{m}\right\}$ with $\lambda_{\varphi}(\{x\}) \geq(1+\sqrt{1-4 \epsilon}) / k$, and we are done. 
Proof of Theorem 3.2. Let $Z$ be a discrete space with $Y=\beta Z$. Since $X$ has $n$ points, say $X:=\left\{x_{1}, \ldots, x_{n}\right\}$, we have that, for each $z \in Z$, $T_{z}$ is of the form $T_{z}:=\sum_{i=1}^{n} a_{i}^{z} \delta_{x_{i}}$, for some $a_{i}^{z} \in \mathbb{K}, i=1, \ldots, n$. Consequently, for each $z \in Z$, we can choose a point $x_{z} \in X$ such that $\left|\lambda_{T_{z}}\left(\left\{x_{z}\right\}\right)\right| \geq\left|\lambda_{T_{z}}(\{x\})\right|$ for every $x \in X$, which yields $\left|\lambda_{T_{z}}\left(\left\{x_{z}\right\}\right)\right| \geq$ $\left\|T_{z}\right\| / n$. This allows us to define a map $h: Z \longrightarrow X$ as $h(z):=x_{z}$ for every $z \in Z$. Since $h$ is continuous we can extend it to a continuous function defined on the whole $Y$, which we also call $h$.

Following a similar process as in the proof of Theorem 2.2, define $\alpha: Z \longrightarrow \mathbb{K}$ as $\alpha(z):=\lambda_{T_{z}}(\{h(z)\})$, and extend it to a continuous function defined on $Y$, also denoted by $\alpha$. Now, define $S: C(X) \longrightarrow$ $C(Y)$ as $(S f)(y):=\alpha(y) f(h(y))$ for every $f \in C(X)$ and $y \in Y$.

Fix any $f \in C(X),\|f\|_{\infty} \leq 1$, and $z \in Z$. It is then easy to check that $|(T f)(z)-(S f)(z)| \leq(n-1)\left\|T_{z}\right\| / n$. Consequently, if $\left\|T_{z}\right\| \leq 2 \sqrt{\epsilon}$, we have

$$
|(T f)(z)-(S f)(z)| \leq \frac{2(n-1)}{n} \sqrt{\epsilon} \leq r_{X}(\epsilon) .
$$

Let us now study the case when $\left\|T_{z}\right\|>2 \sqrt{\epsilon}$. First, we know from $[1$, Corollary 2.5] that $|(T f)(z)-(S f)(z)| \leq\left\|T_{z}\right\|-\sqrt{\left\|T_{z}\right\|^{2}-4 \epsilon}$. Next, we split the proof into two cases.

- Case 1. Suppose that $n$ is odd. We see that to finish the proof it is enough to show that

$$
\min \left(\left\|T_{z}\right\|-\sqrt{\left\|T_{z}\right\|^{2}-4 \epsilon}, \frac{n-1}{n}\left\|T_{z}\right\|\right) \leq r_{X}(\epsilon)
$$

whenever $\left\|T_{z}\right\|>2 \sqrt{\epsilon}$. To do this, we consider the functions $\gamma, \delta:[2 \sqrt{\epsilon}, 1] \longrightarrow \mathbb{R}$ defined respectively as $\gamma(t):=t-\sqrt{t^{2}-4 \epsilon}$, and $\delta(t):=(n-1) t / n$ for every $t \in[2 \sqrt{\epsilon}, 1]$. We have that $\gamma$ is decreasing (see [1, Lemma 3.4]) and $\delta$ is increasing on the whole interval of definition.

Now, if $\epsilon \leq \omega_{n}$, then for $t_{0}:=\sqrt{\epsilon / \omega_{n}} \in[2 \sqrt{\epsilon}, 1]$, we have $\gamma\left(t_{0}\right)=\delta\left(t_{0}\right)$. This common value turns out to be $\delta\left(t_{0}\right)=$ $2 \sqrt{(n-1) \epsilon /(n+1)}$, that is, it is equal to $r_{X}(\epsilon)$, and we get that $|(T f)(z)-(S f)(z)| \leq r_{X}(\epsilon)$ for every $z \in Z$.

On the other hand, if $\epsilon>\omega_{n}$, then $\delta(1) \leq \gamma(1)$, so $\delta(t) \leq \gamma(t)$ for every $t \in[2 \sqrt{\epsilon}, 1]$, and $|(T f)(z)-(S f)(z)| \leq \delta(1)$ for every $z \in Z$. Since $\delta(1)=(n-1) / n=r_{X}(\epsilon)$, we obtain the desired inequality also in this case. 
- Case 2. Suppose that $n$ is even. By Proposition 3.3, we get that

$$
\left|\lambda_{T_{z}}(\{h(z)\})\right| \geq\left(\left\|T_{z}\right\|+\sqrt{\left\|T_{z}\right\|^{2}-4 \epsilon}\right) / n,
$$

So

$$
|(T f)(z)-(S f)(z)| \leq\left\|T_{z}\right\|-\frac{\left\|T_{z}\right\|+\sqrt{\left\|T_{z}\right\|^{2}-4 \epsilon}}{n} .
$$

Consequently, to finish the proof in this case we just need to show that

$$
\min \left(\left\|T_{z}\right\|-\sqrt{\left\|T_{z}\right\|^{2}-4 \epsilon},\left\|T_{z}\right\|-\frac{\left\|T_{z}\right\|+\sqrt{\left\|T_{z}\right\|^{2}-4 \epsilon}}{n}\right) \leq \frac{2(n-1) \sqrt{\epsilon}}{n} .
$$

Let $\eta:[2 \sqrt{\epsilon}, 1] \longrightarrow \mathbb{R}$ be defined as

$$
\eta(t):=t-\frac{t+\sqrt{t^{2}-4 \epsilon}}{n}
$$

for every $t \in[2 \sqrt{\epsilon}, 1]$, and consider also the function $\gamma$ defined above. Clearly, when $n=2$ we have $\eta=\gamma / 2$, and the above inequality follows from [1, Lemma 3.4]. So we assume that $n \neq$ 2. We easily see that $\eta(t) \leq \gamma(t)$ whenever $t \in\left[2 \sqrt{\epsilon}, \sqrt{\epsilon / \omega_{n-1}}\right]$, and that $\eta$ is decreasing in $\left[2 \sqrt{\epsilon}, \sqrt{\epsilon / \omega_{n-1}}\right](t \leq 1)$. We deduce that

$$
\min (\gamma(t), \eta(t)) \leq \eta(2 \sqrt{\epsilon})=\frac{2(n-1) \sqrt{\epsilon}}{n}
$$

whenever $2 \sqrt{\epsilon} \leq t \leq 1$, as it was to be seen.

By denseness of $Z$ in $Y$, we conclude that $\|T-S\| \leq r_{X}(\epsilon)$.

\section{REFERENCES}

[1] J. Araujo and Juan J. Font, Stability of weighted composition operators between spaces of continuous functions. J. London Math. Soc (2) 79 (2009), 363-376.

[2] J. Araujo and Juan J. Font, Stability and instability of continuous linear functionals. Submitted.

[3] V. I. Bogachev, Measure Theory, Vol. II. Springer, 2007.

[4] G. Dolinar, Stability of disjointness preserving mappings. Proc. Amer. Math. Soc. 130 (2002), 129-138.

[5] J. J. Font and S. Hernández, On separating maps between locally compact spaces. Arch. Math. (Basel) 63 (1994), 158-165. 
[6] K. Jarosz, Automatic continuity of separating linear isomorphisms. Canad. Math. Bull. 33 (1990), 139-144.

[7] J.-S. Jeang and N.-C. Wong, Weighted composition operators of $C_{0}(X)$ 's. J. Math. Anal. Appl. 201 (1996), 981-993.

Departamento de Matemáticas, Estadística y Computación, Universidad de Cantabria, Facultad de Ciencias, Avda. de los Castros, s. N., E-39071 SANTANDER, SPAIN

E-mail address: araujoj@unican.es

Departamento de Matemáticas, Universitat Jaume I, Campus Riu Sec, 8029 AP, CASTEllón, Spain

E-mail address: font@mat.uji.es 